\title{
Antithrombotic Properties in Rabbits of Heparin and Heparin Fragments Covalently Coupled to Human Antithrombin III
}

Christer Mattsson, Marc Hoylaerts, Erik Holmer, Turid Uthne, and Désiré Collen

KabiVitrum AB, Research and Development S-1128, Stockholm, Sweden, and Center for Thrombosis and Vascular Research, Department of Medical Research, University of Leuven B-3000, Belgium

\begin{abstract}
Clinical grade heparin is a very heterogeneous mucopolysaccharide, containing molecules with $M_{\mathrm{r}}$ ranging from 6,000 to 30,000 that have either a high affinity or a low affinity for antithrombin III (AT). In this study, the antithrombotic properties of intact high-affinity heparin $\left(M_{r}=15,000\right)$ and of two heparin fragments $\left(h_{16}\right.$, a 16-monosaccharide fragment, with $M_{\mathrm{r}}=4,300$, and $h_{12}$, a 12-monosaccharide fragment, with $M_{\mathrm{r}}$ $=3,200$ ) and of their functional covalent stoichiometric complexes with human AT were compared in a venous thrombosis stasis model in rabbits. Thrombosis was induced by injection of glass-activated human plasma and measured in a segment of the jugular vein that was isolated between two vascular clamps for $10 \mathrm{~min}$.

Injections of $55 \mu \mathrm{g} / \mathrm{kg}$ resulted in a clear antithrombotic effect for intact heparin, but not for the two fragments. Equivalent amounts (carbohydrate moiety) of covalent complexes of heparin or of both heparin fragments with human AT resulted in an antithrombotic effect lasting for 45-60 min. Injection of $110 \mu \mathrm{g} / \mathrm{kg}$ of heparin and of the heparin fragments yielded an antithrombotic effect, lasting $45-60 \mathrm{~min}$; the corresponding amounts of covalent complexes caused an antithrombotic effect for $60-120 \mathrm{~min}$. The free and conjugated fragments produced equal antithrombotic effects at equal plasma levels of anti-Factor $\mathrm{Xa}$ activity, but the specific antithrombotic activities of free and complexed intact heparin, on a molar basis, were 10-20-fold greater than those of the free and complexed heparin fragments. The plasma half-life of the covalent complexes of the heparin fragments with AT is, however, 10 times longer than that of the complex between intact heparin and AT and 30 times longer than that of free intact heparin. Covalent complexes between AT and heparin fragments could, therefore, be useful to maintain more stable levels of antithrombotic activity in plasma.
\end{abstract}

\section{Introduction}

The anticoagulant activity of heparin is due to catalysis of the inhibition of several activated coagulation factors by the plasma protease inhibitor antithrombin III $(\mathrm{AT})^{1}(1,2)$. Active heparin

Received for publication 24 August 1984 and in revised form 18 December 1984

1. Abbreviations used in this paper: AT, antithrombin III; $\mathrm{H}$, highaffinity heparin of average $M_{\mathrm{r}}=15,000 ; \mathrm{h}_{12}$, high-affinity heparin fragment of $M_{\mathrm{r}}=3,200$, containing an average of 12 monosaccharide units; $h_{16}$, high-affinity heparin fragments of $M_{r}=4,300$, containing an average of 16 monosaccharides; $k$, second order rate constant(s).

J. Clin. Invest.

(c) The American Society for Clinical Investigation, Inc.

0021-9738/85/04/1169/05 \$1.00

Volume 75, April 1985, 1169-1173 molecules contain a unique pentasaccharide sequence that binds with high affinity to AT (3); in clinical grade heparin preparations, this sequence is only present in about one-third of the molecules (4). In addition, clinical grade heparin is very heterogeneous with respect to $M_{\mathrm{r}}$, ranging from 6,000 to 30,000 with an average of $10,000-15,000(5)$.

High-affinity heparin fractions of low $M_{\mathrm{r}}$ retain the ability to catalyze the inhibition of some coagulation factors, among them Factor Xa, while having a markedly reduced effect on the neutralization of other coagulation enzymes, among them thrombin. Such low- $M_{\mathrm{r}}$ heparins have high anti-Factor Xa activity but do not significantly prolong the activated partial thromboplastin time (6-8).

It has been suggested that heparin fractions of low $M_{\mathrm{r}}$ and with a high anti-Factor $\mathrm{Xa}$ /activated partial thromboplastin time activity ratio still show antithrombotic activity but are associated with a reduced incidence of bleeding $(9,10)$. This was recently demonstrated in rabbits with experimental venous thrombosis, using heparin fragments obtained by controlled nitrous acid degradation $(11,12)$. In addition, the antithrombotic activity of different heparin fragments not only depends on their specific anticoagulant activity, but it also seems to be a function of the chain length of the fragment (11). Furthermore, it has been shown that low-affinity heparin potentiates the antithrombotic effect of high-affinity heparin fragments (13).

Complex formation between heparin and AT is an essential step in the mechanism of inhibition of activated coagulation factors $(2,7,14)$. Recently, complexes between heparin and AT have been stabilized by covalently coupling AT to heparin or to heparin fragments $(15,16)$. These covalent complexes inhibit Factor $\mathrm{Xa}$ and thrombin with second order rate constants $(k)$ very similar to those found for the corresponding reversible complexes (15-17).

In this study, the antithrombotic properties of covalent complexes between AT and heparin or heparin fragments were compared with those of free high-affinity heparin or highaffinity heparin fragments in an experimental venous thrombosis model in rabbits.

\section{Methods}

Materials. Human AT was purified from plasma by affinity chromatography on heparin Sepharose (18). Protein concentration was determined by absorbance measurements using an extinction coefficient, $\mathrm{A}_{\mathrm{lcm}}^{\mathrm{iq}}(280 \mathrm{~nm})=6.2$, and an $M_{\mathrm{r}}$ of $56,000(18)$.

High-affinity heparin of average $M_{\mathrm{r}}=15,000(\mathrm{H})$ was purified from clinical grade heparin (Kabi AB, Stockholm, Sweden) by affinity chromatography on matrix-bound AT (4). High-affinity heparin fragments of $M_{\mathrm{r}}=4,300$, containing an average of 16 monosaccharides $\left(h_{16}\right)$ and of 3,200, containing an average of 12 monosaccharide units $\left(h_{12}\right)$ were prepared by partial deaminative cleavage of heparin with nitrous acid $(19,20)$ and subsequent affinity chromatography on AT Sepharose. In this study only high-affinity heparin fragments purified on AT Sepharose were used.

The $M_{\mathrm{r}}$ of the fragments was determined by gel filtration on 
Sephadex G75 (Pharmacia, Uppsala, Sweden) (11). The characteristics and homogeneity of the fragments were very similar to those described by Thomas et al. (21): $h_{16}$ contained polysaccharides ranging from 14 to 18 monosaccharide units, and $h_{12}$ consisted of material with 10 to 14 monosaccharide units. Heparin concentrations were measured using the carbazole- $\mathrm{H}_{2} \mathrm{SO}_{4}$ method (22), and heparin activity was determined by Factor $\mathrm{Xa}$ inhibition using the chromogenic substrate $N$-benzoyl-Lisoleucyl-L-glutamyl-glycyl-L-arginine-pNA (S-2222; Kabi AB) (6).

Covalent complexes between AT and heparin or heparin fragments were synthesized as previously described $(15,16)$. Primary amino groups were introduced in the mucopolysaccharide through reaction with hexamethylenediamine, and the fraction of the substituted heparin having a high affinity for AT was linked to AT with the bifunctional reagent tolylene-2,4-diisothiocyanate. The covalent complexes were purified by chromatography on DEAE-Sephacel (Pharmacia). The ATH complex was further purified by gel filtration on Ultrogel AcA 44 (LKB Produkter, Bromma, Sweden) in 0.1 M Tris $\mathrm{HCl}$ buffer, $\mathrm{pH}$ 7.6. The purified complexes were quantified by the carbazole- $\mathrm{H}_{2} \mathrm{SO}_{4}$ method (heparin moiety) and by radial immunodiffusion using antibodies against human AT in the gel. Both determinations agreed within $10 \%$. Carbazole measurements were not influenced by the presence of AT. The AT concentration in the complexes, as determined by titration against thrombin, was $>85 \%$ of that estimated by the method of Lowry et al. (23). The disappearance of anticoagulant activity from the plasma in rabbits in vivo was followed by measuring Factor $\mathrm{Xa}$ inhibition in a plasma medium $(11,24,25)$.

The antithrombotic properties of the heparin or heparin fragments and of their corresponding covalent complexes with AT were evaluated in a rabbit thrombus model originally described by Wessler et al. (26) and previously applied to compare the antithrombotic effect of intact heparin and heparin fragments (11). The model involves activation of the rabbit's coagulation system by intravenous injection of glassactivated human plasma. New Zealand white rabbits weighing 2.5-3.5 $\mathrm{kg}$ were premedicated with atropine $(0.1 \mathrm{mg} / \mathrm{kg})$ and Hypnorm ${ }^{\oplus}(0.1$ $\mathrm{ml} / \mathrm{kg}$ ) (Leo AB, Helsingborg, Sweden) and then anesthetized with $2 \%$ pentobarbitone. After injection of $1.5 \mathrm{ml} / \mathrm{kg}$ of glass-activated human plasma, a 2-cm-long segment of the jugular vein (containing 0.2-0.3 $\mathrm{ml}$ blood) was isolated between two vessel clamps. The isolated vein segment was removed after 10 min of stasis, its contents were emptied into a petri dish containing $0.9 \% \mathrm{NaCl}$, and both the size and appearance of the formed clot(s) were scored on a scale graded from 0 to 4 (26). A score of 4 was given for a single large thrombus forming a cast of the isolated segment, 3 for two or more large thrombi, 2 for several small thrombi, 1 for a few macroscopic strands of fibrin, and 0 for fluent blood. The antithrombotic effect was tested 15,30 , and 45 min after injection of the test compounds. The covalent complexes between AT and $h_{16}$ or $h_{12}$ with a longer duration of the antithrombotic effect were also tested after 60,90 , and $120 \mathrm{~min}$.

A minimum of four separate determinations was made for each time point and test compound; when results were too variable another two to three experiments were performed. About every fifth rabbit served as a control injected with saline only. The in vivo applications and the scoring of thrombi were carried out by an investigator unaware of the test compound that was injected.

For each test compound, a mean antithrombotic score was calculated by averaging the individual data for each time point. A score below 2 was considered to represent a significant antithrombotic effect; a score between 2 and 3, a moderate effect; and scores above 3, no antithrombotic activity. Differences in anticoagulant activity were statistically analyzed using $t$ test.

Immediately before the injection of the thrombogenic plasma, 1.8$\mathrm{ml}$ blood samples were collected from a cannulated ear artery and mixed with $200 \mu \mathrm{l}$ of $10 \%$ sodium citrate for measurement of antiFactor Xa activity.

Anti-Factor Xa activity was measured in a plasma environment using the chromogenic substrate S-2222. Details of the assay and its calibration are reported elsewhere (11)

\section{Results}

Table I summarizes the specific anticoagulant activities of the free heparin moieties and of their corresponding AT-heparin complexes, as determined by the anti-Factor Xa assay. The specific activities of the complexes are very similar to those of the corresponding free fragments both for AT-H and for AT$h_{12}$. By repeated assays, the specific activity of $A T-h_{16}$ appears to be somewhat higher than that of the free fragment. This difference is larger than can be explained by experimental error, because the anti-Factor $\mathrm{Xa}$ assay has a coefficient of variation of $10-15 \%$.

The antithrombotic effect of the free and complexed heparins is illustrated in Fig. 1. $15 \mathrm{~min}$ after an intravenous injection of $55 \mu \mathrm{g} / \mathrm{kg}$, the mean thrombosis scores for $\mathrm{H}$ and AT-H were 1.7 and 0.7 , respectively $(n=6)$. Corresponding values after $30 \mathrm{~min}$ were 3.4 and 2.3 (Fig. $1 \mathrm{~A}$ ). The results suggest a somewhat higher antithrombotic effect or a prolonged duration of the effect for the complex as compared with the free heparin. Intravenous injection of $55 \mu \mathrm{g} / \mathrm{kg}$ of $h_{16}$ or $h_{12}$ did not produce an antithrombotic effect, whereas equivalent amounts of their covalent complexes were active (Fig. 1, C and $E$ ).

At a dose of $110 \mu \mathrm{g} / \mathrm{kg}$ a significantly higher antithrombotic effect was obtained as evidenced by lower mean thrombosis scores. A prolonged duration of the antithrombotic effect is observed for all heparin substances tested. $15 \mathrm{~min}$ after administration, both for $\mathrm{H}$ and $\mathrm{AT}-\mathrm{H}$, zero scores were observed, corresponding to full protection, while scores after $30 \mathrm{~min}$ averaged 1.2 and 1.0, both for $\mathrm{H}$ and $\mathrm{AT}-\mathrm{H}$, respectively (Fig. $1 B$ ). For the free fragments $h_{16}$ and $h_{12}$, injection of $110 \mu \mathrm{g} /$ $\mathrm{kg}$ yielded a significant antithrombotic effect (Fig. 1, D and $F)$. After $45 \mathrm{~min}$, however, the protection by $h_{16}$ and $h_{12}$ disappeared, while, on the contrary, for AT- $h_{16}$ and AT- $h_{12}$ it took $120 \mathrm{~min}$ and $90 \mathrm{~min}$, respectively (Fig. 1, D and F), before the antithrombotic protection disappeared.

The disappearance rate of the anti-Factor Xa activity from the plasma of the rabbits after injection of $110 \mu \mathrm{g} / \mathrm{kg}$ of the free or complexed heparin moieties is shown in Fig. 2. Because the different heparin substances have different specific activities (Table I) and because an equal dose of $110 \mu \mathrm{g} / \mathrm{kg}$ was given in all cases, different initial anti-Factor $\mathrm{Xa}$ activities were obtained in the blood. Initially, the anticoagulant activity disappeared rapidly, reaching $50 \%$ after $9 \mathrm{~min}$ for $\mathrm{H}, 18 \mathrm{~min}$

Table I. Specific Anti-Factor Xa Activity of the High-Affinity Heparins and of Their Covalent Complexes with AT

\begin{tabular}{ll}
\hline Heparin substance & Heparin moiety \\
\hline & $I U / m g$ \\
$\mathrm{H}$ & 300 \\
$\mathrm{AT}-\mathrm{H}$ & 270 \\
$\mathrm{~h}_{16}$ & 540 \\
$\mathrm{AT}-\mathrm{h}_{16}$ & 850 \\
$\mathrm{~h}_{12}$ & 860 \\
AT- $\mathrm{h}_{12}$ & 760
\end{tabular}

AT-H, AT- $h_{16}$, and AT- $h_{12}$, covalent complexes between AT and H, $h_{16}$, or $h_{12}$. 

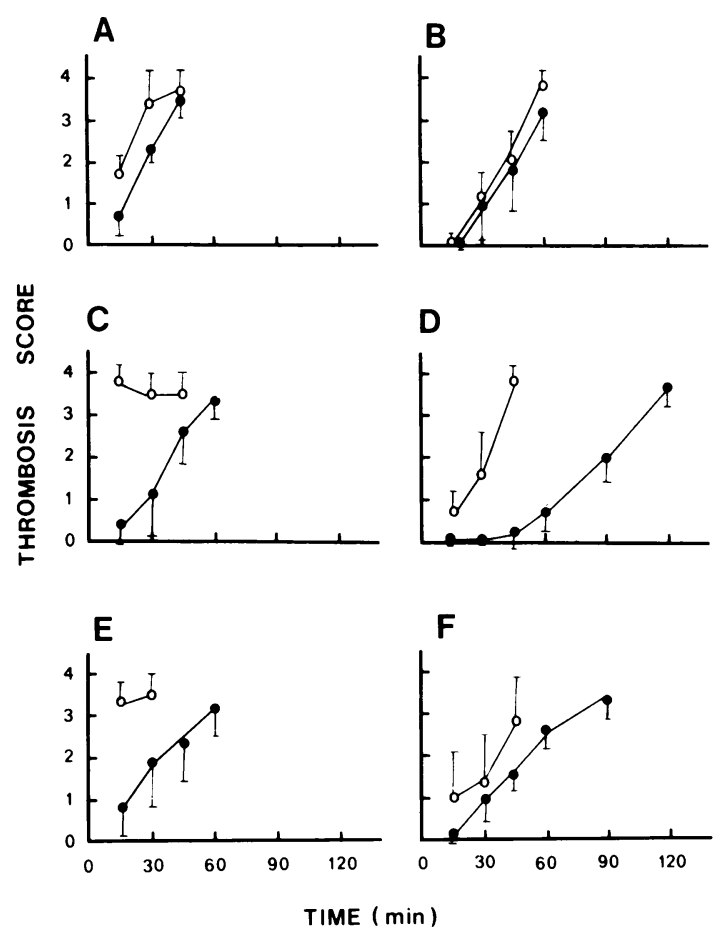

Figure 1. Antithrombotic effect of intact $\mathrm{H}(A, B)$ and of $\mathrm{h}_{16}(C, D)$ and $\mathrm{h}_{12}(E, F)$ in a venous thrombosis model in rabbits. $O$, free heparin moieties; $\bullet$, covalent complexes between the heparin moieties and AT. $A, C$, and $E, 55 \mu \mathrm{g} / \mathrm{kg}$ dose; $B, D$, and $F, 110 \mu \mathrm{g} / \mathrm{kg}$ dose. The data represent mean \pm SD of at least four experiments.

for AT-H, $53 \mathrm{~min}$ for $\mathrm{h}_{16}, 80 \mathrm{~min}$ for AT-h $16,50 \mathrm{~min}$ for $\mathrm{h}_{12}$, and $85 \mathrm{~min}$ for $\mathrm{AT}-\mathrm{h}_{12}$.

Table II represents the duration of the antithrombotic effect, measured as the time to reach an antithrombotic score of 2. This duration varied from $18 \mathrm{~min}(55 \mu \mathrm{g} / \mathrm{kg} \mathrm{H})$ to 90 $\min \left(110 \mu \mathrm{g} / \mathrm{kg} \mathrm{AT}-\mathrm{h}_{16}\right)$. The anti-Factor Xa levels in plasma measured at the time of the thrombosis score 2 are also represented in Table II. These data show that the anti-Factor $\mathrm{Xa}$ levels at score 2 were similar for free and conjugated

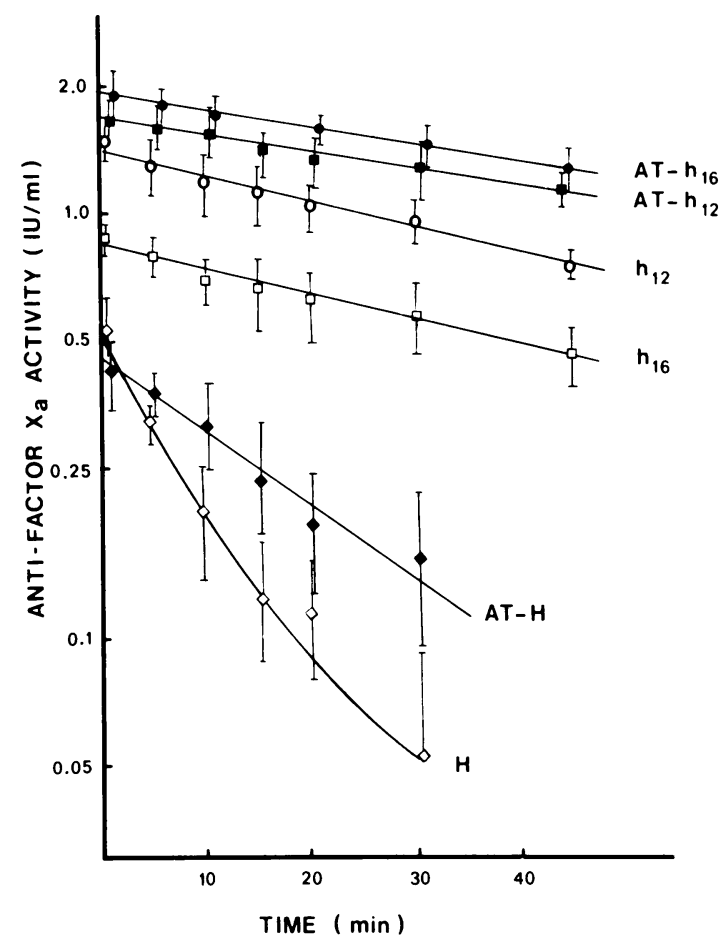

Figure 2. Initial disappearance rate of anti-Factor Xa activity after intravenous injection into rabbits of $110 \mu \mathrm{g} / \mathrm{kg}$ free or complexed heparin moieties.

heparin moieties but very different for $\mathrm{H}$ as compared with $h_{16}$ and $h_{12}$.

\section{Discussion}

The antithrombotic effect of intact $\mathrm{H}$ and of $h_{16}$ and $h_{12}$ was investigated in a venous thrombosis model in rabbits. Both the free heparin moieties and their covalent complexes with AT were studied. At doses of $55 \mu \mathrm{g} / \mathrm{kg}$ only free $\mathrm{H}$ but not free $h_{16}$ or $h_{12}$ had a significant antithrombotic effect, notwithstanding the fact that the anti-Factor Xa activity in plasma

Table II. Duration of the Antithrombotic Effect After Intravenous Injection of High-Affinity Heparin (Fragments) or AT-Heparin (Fragment) Complexes in Rabbits

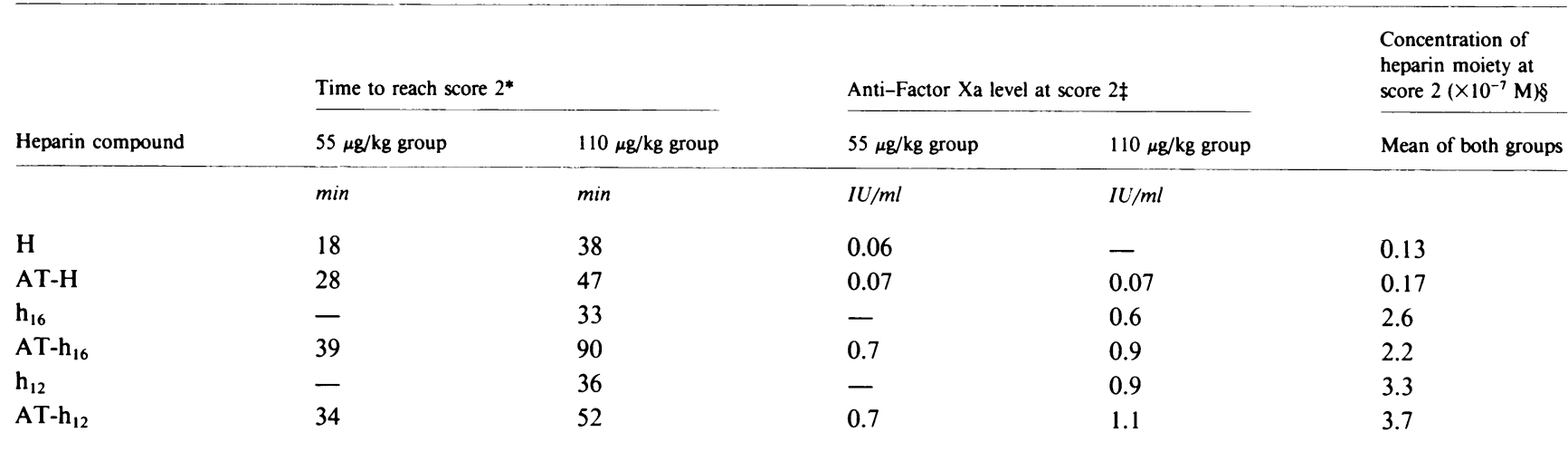

* Derived from Fig. 1. $¥$ Derived from anti-Factor Xa assays on serial blood samples after injection of the heparin moieties (e.g., Fig. 2). $\S$ Obtained as the quotient of the anti-Factor Xa level at the time of score 2 and the specific anti-Factor Xa activity of the heparin moieties expressed in molar concentration. 
after injection of the fragments was two- to fourfold greater than that obtained with $\mathrm{H}$. In previous studies (11) it has been shown that heparin fragments with molecular weights of $\sim 4,000$ are equipotent to heparin with respect to antithrombotic effects. Barrowcliffe et al. (13), however, demonstrated that low-affinity forms of heparin potentiate the antithrombotic effect of high-affinity forms. At a dose of $110 \mu \mathrm{g} / \mathrm{kg}, \mathrm{h}_{16}$ and $h_{12}$ exerted an antithrombotic effect that lasted until the antiFactor Xa level had decreased by $\sim 50 \%$.

The covalent complex between AT and $\mathrm{H}$ had a slightly more pronounced antithrombotic effect than free $\mathrm{H}$, a result that agrees with the complex's two- to threefold longer halflife (15) (Fig. 2). The covalent complexes between AT and $h_{16}$ and $h_{12}$ had a significantly greater antithrombotic effect than the free fragments (Fig. 1), which may be explained by the finding that for equivalent amounts injected, the level of antiFactor Xa activity in plasma was higher for the complexes than for the free fragments (Fig. 2). In addition, the complexes disappeared more slowly from the circulation.

To compare the specific antithrombotic activities of the free heparin moieties and their covalent complexes, the Factor $\mathrm{Xa}$ inhibition levels were calculated when the antithrombotic activity in plasma had reached an intermediate level (score 2). This occurred at a concentration of $0.5-1.0 \mathrm{IU} / \mathrm{ml}$, both for the free and complexed forms of $h_{16}$ and $h_{12}$ (corresponding to concentrations of $0.2-0.4 \mu \mathrm{M}$ ), but at a 10-fold lower concentration for free or complexed $\mathrm{H}$, indicating that the free and complexed heparin moieties have similar specific antithrombotic activities. The specific antithrombotic activity of $\mathrm{H}$, however, was more than an order of magnitude greater than that of $h_{16}$ or $h_{12}$, as expressed in both molar concentration and anti-Factor Xa activity, indicating that the antithrombotic effect of heparin in plasma does not only depend on its antiFactor Xa activity. This lack of correlation between the antithrombotic and anti-Factor $\mathrm{Xa}$ activity of heparin fragments confirms previous reports $(21,27)$.

The different ratios between anti-Factor Xa activity and antithrombotic efficacy of $\mathrm{H}$ as compared with $h_{16}$ or $h_{12}$ may be due to differences in their kinetics of inhibition of thrombin and Factor Xa. Thus AT-H inhibits Factor Xa with a $k$ of 2 $\times 10^{6} \mathrm{M}^{-1} \mathrm{~s}^{-1}(15)$ and thrombin with a $k$ of $3 \times 10^{8} \mathrm{M}^{-1} \mathrm{~s}^{-1}$ (17). AT-h $\mathrm{h}_{16}$ inhibits Factor Xa with a $k$ of $1.3 \times 10^{6} \mathrm{M}^{-1} \mathrm{~s}^{-1}$ and thrombin with a $k$ of $2 \times 10^{7} \mathrm{M}^{-1} \mathrm{~s}^{-1}$, whereas AT- $\mathrm{h}_{12}$ inhibits Factor Xa with a $k$ of $10^{6} \mathrm{M}^{-1} \mathrm{~s}^{-1}$ and thrombin with a $k$ of $3 \times 10^{5} \mathrm{M}^{-1} \mathrm{~s}^{-1}$ (15-17). Thus AT-H inhibits thrombin 100 times faster than Factor Xa. At a concentration of AT-H of $17 \mathrm{nM}$ (score 2, Table II) thrombin will be inhibited with a $t_{1 / 2}=0.5 \mathrm{~s}$ and Factor Xa with a $t_{1 / 2}=20 \mathrm{~s}$. Likewise, at a concentration of AT- $h_{16}$ of $220 \mathrm{nM}$ (score 2, Table II), thrombin will be inhibited with a $t_{1 / 2}=0.5 \mathrm{~s}$ and Factor $\mathrm{Xa}$ with a $t_{1 / 2}=2.5 \mathrm{~s}$. At a concentration of AT- $\mathrm{h}_{12}$ of $370 \mathrm{nM}$, the half-life of thrombin will be $7 \mathrm{~s}$ and that of Factor $\mathrm{Xa}, 2$ s. These half-life values are probably an underestimate of the real half-lives, because the free AT-H concentration is lower than the total concentration (28). They do, however, suggest that the antithrombotic effect of AT-H (and of $\mathrm{H}$ ) is probably mainly due to its antithrombin activity, whereas that of AT$h_{16}$ and AT-h $h_{12}$ (and of $h_{16}$ and $h_{12}$ ) seems to be due to their anti-Factor Xa activity.

The present study was carried out with the lowest concentrations of heparin moieties and their complexes, which resulted in a clear antithrombotic effect that disappeared when the plasma concentration decreased two- to fourfold. Under the experimental conditions used, the disappearance of the anticoagulant activity is thus due both to turnover of the antithrombotic substances and to their equilibration with the extravascular space. The half-life in plasma after equilibration is, however, two- to threefold longer for the covalent complexes than for the free forms $(15,16)$, and 10 -fold longer for $h_{16}$ and $h_{12}$ than for $H$ (16). The half-life of AT- $h_{16}$ and AT- $h_{12}$ is 30 fold longer than that of $\mathrm{H}$ and 10-fold longer than that of ATH. Thus, even with a 10-fold lower specific antithrombotic activity, AT- $h_{16}$ and AT- $h_{12}$ complexes could produce a stable antithrombotic state, requiring smaller amounts than AT-H; discontinuous injections would result in more stable plasma levels, and far less frequent injections would be required to maintain an antithrombotic concentration in plasma. Covalent complexes between AT and $h_{16}$ or $h_{12}$ could, therefore, be of potential advantage as antithrombotic agents in humans.

\section{Acknowledgments}

This study was supported by project $80-8513$ of the Geconcerteerde Ohderzoeksacties.

\section{References}

1. Björk, I., and B. Nordenman. 1976. Acceleration of the reaction between thrombin and antithrombin III by non-stoichiometric amounts of heparin. Eur. J. Biochem. 68:507-511.

2. Jordan, R., D. Beeler, and R. D. Rosenberg. 1979. Fractionation of low molecular weight heparin species and their interaction with antithrombin. J. Biol. Chem. 254:2902-2913.

3. Lindahl, U., G. Bäckström, and L. Thunberg. 1983. The antithrombin-binding sequence of heparin. Identification of an essential 6-O-sulphate group. J. Biol. Chem. 258:9826-9830.

4. Andersson, L.-O., T. W. Barrowcliffe, E. Holmer, E. A. Johnson, and G. E. C. Sims. 1976. Anticoagulant properties of heparin fractionated by affinity chromatography on matrix-bound antithrombin III and by gel filtration. Thromb. Res. 9:575-583.

5. Ehrlich, J., and S. S. Stivala. 1973. Chemistry and pharmacology of heparin. J. Pharm. Sci. 62:527-544.

6. Andersson, L.-O., T. W. Barrowcliffe, E. Holmer, E. A. Johnson, and G. Söderström. 1979. Molecular weight dependency of the heparin potentiated inhibition of thrombin and activated factor X. Effect of heparin neutralization in plasma. Thromb. Res. 15:531-541.

7. Thunberg, L., U. Lindahl, N. Tengblad, T. C. Laurent, and C. M. Jackson. 1979. On the molecular-weight-dependence of the anticoagulant activity of heparin. Biochem. J. 181:241-243.

8. Holmer, E., K. Kurachin, and G. Söderström. 1981. The molecular weight dependence of the rate-enhancing effect of heparin on the inhibition of thrombin, Factor Xa, Factor IXa, Factor XIa, Factor XIIa and Kallikrein by antithrombin. Biochem. J. 193:395400.

9. Holmer, E. 1980. Anticoagulant properties of heparin and heparin fractions. Scand. J. Haematol. 25(Suppl. 36):25-39.

10. Thomas, D. P., T. W. Barrowcliffe, and E. A. Johnson. 1980. The influence of tissue source, salt and molecular weight on heparin activity. Scand. J. Haematol. 25(Suppl. 36):40-49.

11. Holmer, E., C. Mattsson, and S. Nilsson. 1982. Anticoagulant and antithrombotic effects of heparin and low molecular weight heparin fragments in rabbits. Thromb. Res. 25:475-485.

12. Carter, C. J., J. C. Kelton, J. Hirsch, A. Cerskus, A. V. Santos, and $M$. Gent. 1982. The relationship between the hemorrhagic and antithrombotic properties of low molecular weight heparin in rabbits. Blood. 59:1239-1245.

13. Barrowcliffe, T. W., R. E. Merton, S. J. Havercroft, L. Thunberg, 
U. Lindahl, and D. Thomas. 1984. Low-affinity heparin potentiates the action of high-affinity heparin oligosaccharides. Thromb. Res. 34: 125-133.

14. Jordan, R. E., G. M. Costa, W. T. Gardner, and R. D. Rosenberg. 1980. The binding of low molecular weight heparin to hemostatic enzymes. J. Biol. Chem. 255:10081-10090.

15. Ceustermans, R., M. Hoylaerts, M. De Mol, and D. Collen. 1982. Preparation, characterization and turnover properties of heparinantithrombin III complexes stabilized by covalent bonds. J. Biol. Chem. 257:3401-3408.

16. Hoylaerts, M., E. Holmer, M. De Mol, and D. Collen. 1983. Covalent complexes between low molecular weight heparin fragments and antithrombin III. Inhibition kinetics and turnover parameters. Thromb. Haemostasis. 49:109-115.

17. Hoylaerts, M., W. G. Owen, and D. Collen. 1984. Involvement of heparin chain length in the heparin-catalyzed inhibition of thrombin by antithrombin III. J. Biol. Chem. 259:5670-5677.

18. Miller-Andersson, M., H. Borg, and L. -O. Andersson. 1974. Purification of antithrombin-III by affinity chromatography. Thromb. Res. 5:439-452.

19. Lindahl, U., G. Bäckström, M. Höök, L. Thunberg, L. A. Fransson, and A. Linker. 1979. Structure of the antithrombin-binding site in heparin. Proc. Natl. Acad. Sci. USA. 76:3198-3202.

20. Choảy, J., J. C. Lormeau, M. Petiton, P. Sinay, B. Casu, P. Oreste, G. Torre, and G. Gatti. 1980. Anti-Xa active heparin oligosaccharides. Thromb. Res. 18:573-578.
21. Thomas, D. P., R. E. Merton, T. W. Barrowcliffe, L. Thunberg, and U. Lindahl. 1982. Effects of heparin oligosaccharides with high affinity for antithrombin III in experimental venous thrombosis. Thromb. Haemostasis. 47:244-248.

22. Bitter, T., and H. M. Muir. 1962. A modified uronic acid carbazole reaction. Anal. Biochem. 4:330-334.

23. Lowry, O. H., N. J. Rosebrough, A. L. Farr, and R. J. Randall. 1951. Protein measurement with the folin phenol reagent. J. Biol. Chem. 193:265-275.

24. Teien, A. N., M. Lie, and U. Abildgaard. 1976. Assay of heparin in plasma using a chromogenic substrate for activated factor X. Thromb. Res. 8:413-416.

25. Teien, A. N., and M. Lie. 1977. Evaluation of an amidolytic heparin assay method: increased sensitivity by adding purified antithrombin III. Thromb. Res. 10:399-410.

26. Wessler, S., S. M. Reimer, and M. C. Sheps. 1959. Biologic assay of a thrombosis-inducing activity in human serum. J. Appl. Physiol. 14:943-946.

27. Ockelford, P. A., C. J. Carter, L. Mitchell, and J. Hirsh. 1982. Discordance between the anti-Xa activity and the antithrombotic activity of an ultra-low molecular weight heparin fraction. Thromb. Res. 28:401-409.

28. Lijnen, H. R., M. Hoylaerts, and D. Collen. 1983. Heparin binding properties of human histidine-rich glycoprotein. Mechanism and role in the neutralization of heparin in plasma. J. Biol. Chem. 258:3803-3808. 